\title{
Sex pheromone of the jumping plant bug, Halticus minutus Reuter (Hemiptera: Miridae)
}

Tao Zhang ${ }^{1}$, Xiaofang Zhang ${ }^{1}$, Xiangdong $\mathrm{Mei}^{2}$, Yaofa $\mathrm{Li}^{1}$, Zhanlin Gao ${ }^{1}$ and Jun $\mathrm{Ning}^{2 *}$

${ }^{1}$ Institute of Plant Protection, Hebei Academy of Agricultural and Forestry Sciences/IPM Centre of Hebei Province, Baoding 071000, People's Republic of China

${ }^{2}$ State Key Laboratory for Biology of Plant Diseases and Insect Pests, Institute of Plant Protection, Chinese Academy of Agricultural Sciences, Beijing 100193, People's Republic of China

Corresponding author:

Jun Ning: jning@ippcaas.cn Tel: 010-62899142

Institute of Plant Protection, Chinese Academy of Agricultural Sciences, No 2 West Yuan Ming Yuan Road, Beijing 100193, China 


\begin{abstract}
The jumping plant bug, Halticus minutus Reuter, is a pest of sweet potatoes, soybeans and turfgrass in China. Here we present our analysis of the female-produced sex pheromone. These plant bugs release their sex pheromone, consisting hexyl acetate (HA), hexyl butyrate (HB) and (E)-4-oxo-2-hexenal (4-OHE), at night. These three components, tested individually, elicited strong antennal responses. Field tests indicated that a ternary blend of HA, HB and 4-OHE, at ratios of 1:16:10, effectively attracted male H.minutus. Our dose-response analysis showed optimal male capture at the trap dose of $13.5 \mathrm{mg} /$ trap. Higher trap doses did not improve capture rates. Higher ratios of HB in the pheromone mix led to substantially reduced captures, from which we infer that HB may function as an alarm pheromone or defensive secretion. Pipette tips were used as long-term lures, and whose longevities were evaluated. Results showed effective duration of pipette tip lures were more than 5 weeks. Thus, we suggest that the pheromone-baited traps are economically viable tools for long-term monitoring and trapping of jumping plant bugs.
\end{abstract}

Key Words_-jumping plant bug (Halticus minutus), sex pheromone, hexyl acetate, hexyl butyrate,

(E)-4-oxo-2-hexenal 


\section{Introduction}

The jumping plant bug, Halticus minutus, is a widespread pest in Asia (Amalin and Vasquez, 1993). Similar to its congeneric species H. bractatus, H. minutus is polyphagous, feeding on a wide recipe, including sweet potato, alfalfa, bean, barley, corn, oat, wheat, eggplant, tobacco, and cotton (Henry, 1983). In China, H. minutus is considered as an important pest in sweet potato and soybean (Tong and Wang, 1987). In recent years, $H$. minutus outbroke in turfgrass in northern China, causing whitish spots on turfgrass leaves (Wang et al., 2005; Xu et al., 2010). In addition to direct feeding, H. minutus, as a leaf-sucking insect, indirectly harmed host plants through transmission of phytoplasmas (Mitchell, 2004). Nowadays, insecticide use is the sole pest control option available, which has caused problems including resistance development, pest resurgence and environmental pollution. Strategies exploiting the chemical communication of $H$. minutus could be an effective and environmentally-friendly management option against this pest.

For decades, insect sex pheromone has been demonstrated to be effective to monitor and control agriculture pests in an environmentally-friendly manner (Witzgall et al., 2010). Although the Miridae are the largest family of Heteroptera, including over 11,000 species (Cassis and Schuh, 2012), the studies and applications of mirid pheromone are still in infant. Up to now, sex pheromones of approximate 20 mirid species have been identified and verified, including Adelphocoris spp. (Zhang et al., 2015; 2016), Apolygus spp. (Yang et al., 2014, 2015), Lygus spp. (Byers et al., 2013; Fountain et al., 2014; Ho and Millar, 2002; Innocenzi et al., 2004, 2005), Phytocoris spp. (Millar and Rice, 1998; Millar et al., 1997; Zhang and 
Aldrich, 2008), and species in other genus (Drijfhout and Groot, 2001; Kakizaki and Sugie, 2001; Lowor et al., 2009; Mahob et al., 2011; Smith et al., 1991; Sarfo et al., 2013; Yasuda et al., 2008;). Most identified communication compounds are saturated or unsaturated straight-chain esters and aldehyde.

As far as we know, there have been no reports published with respect to the chemical ecology of genus Halticus. The aim of our research is to identify potential sex pheromone components, to optimum their active ratios, and to further evaluate the activities of artificial lures in the field. This study could help us to decipher chemical communication of Halticus genus, and develop novel strategies to effectively monitor and control H. minutus. 


\section{Materials and Methods}

\section{Insects and Sample Collection}

Nymphs of Halticus minutus were collected by sweep-netting from a lawn in Institute of Plant Protection, Chinese Academy of Agricultural Sciences, Beijing, and subsequently cultured at $25 \pm 2{ }^{\circ} \mathrm{C}, 50 \% \sim 70 \% \mathrm{RH}$, and $12: 12$ (L:D) photoperiod in environmental growth chambers (Ningbo Jiangnan Instrument, Ningbo, China). The nymphs were reared on fresh turfgrass. Newly-emerged bugs were sorted by gender according to the ovipositor in female abdomen.

Emitted volatiles were collected using the aeration method described previously (Innocenzi et al., 2004). Briefly, volatiles from single virgin adult ( 3-day-old) were drawn from a glass chamber $(30 \mathrm{~cm}$ height $\times 3.5 \mathrm{~cm}$ diam $)$ using a diaphragm pump $\left(300 \mathrm{~mL} \cdot \mathrm{min}^{-1}\right)$ and adsorbed on a glass tube $(0.5 \mathrm{~cm}$ diam) containing $50 \mathrm{mg}$ of purified PorapakQ. Ten duplicates for both genders were conducted individually. All samples were collected for the first 2 hours of the scotophase. Then, adsorbed aeration volatiles were eluted from PorapakQ with $500 \mu \mathrm{L}$ of distilled dichloromethane. The extracts were stored at $-20^{\circ} \mathrm{C}$ before GC-MS measurement.

\section{GC-MS Analysis}

Collections of volatiles emitted from $H$. minutus were analyzed with a gas chromatography-mass spectrometer (7890A-5975C, Agilent, Santa Clara, California) equipped with a DB-wax $(30 \mathrm{~m} \times 0.25 \mathrm{~mm} \times 0.25 \mu \mathrm{m}$, film thickness; Agilent $)$ or DB-5 column 
$(30 \mathrm{~m} \times 0.25 \mathrm{~mm} \times 0.25 \mu \mathrm{m}$, film thickness; Agilent $)$. The carrier gas was helium in $1.0 \mathrm{~mL} \cdot \mathrm{min}^{-1}$. The oven temperature was held at $50^{\circ} \mathrm{C}$ for $1 \mathrm{~min}$ and then programmed at $3^{\circ} \mathrm{C} \cdot \mathrm{min}^{-1}$ to $120^{\circ} \mathrm{C}$; then at $20^{\circ} \mathrm{C} \cdot \mathrm{min}^{-1}$ to $250^{\circ} \mathrm{C}$ for $5 \mathrm{~min}$. The MS operating conditions were as follows: transfer line temperature, $250^{\circ} \mathrm{C}$; ion source temperature, $230^{\circ} \mathrm{C}$; ionization energy, $70 \mathrm{eV}$; and scan range m/z. 50-400. Mass spectra were matched to those in the NIST14 database, and the identifications were confirmed by comparing retention indices and spectra with those of authentic standards. Retention indices were calculated by the retention times of target compound relative to those of $n$-alkanes. External standard curves, obtained from series concentration of each authentic compound, were used for quantitative analysis of pheromone compounds.Electroantennography

EAG apparatus (Syntech Ltd., Hilversum, Netherlands) was used in electroantennography assay to evaluate whether the identified compounds stimulate $H$. minutus using a previous method (Sun et al., 2014). In brief, the amputated antennae of $H$. minutus males ( 3-days-old) with excision of tips were immediately attached to two electrodes of fork holder with electrically conductive gel (Syntech). Tested compounds were dissolved into liquid paraffin, and $10 \mu \mathrm{L}$ of serial dilutions $\left(10,1,0.1,0.01\right.$ and $\left.0.001 \mu \mathrm{g} \cdot \mu \mathrm{L}^{-1}\right)$ were then added to a folded filter paper, which was inserted into a glass Pasteur pipette. An air stimulus controller CS-55 was used for air and chemical stimulant delivery to the antennae with a constant flow of $150 \mathrm{~mL} \cdot \mathrm{min}^{-1}$ at $30 \mathrm{~s}$ intervals. Liquid paraffin was tested as a background and standard stimulus.

\section{Chemicals}


Hexyl acetate (98\% purity) and hexyl butyrate (95\% purity) were obtained from commercial sources (ABCR GmbH \& CO. KG), (E)-4-Oxo-2-hexenal was synthesized as described by Moreira and Millar (2005). The purity of synthetic (E)-4-Oxo-2-hexenal was $>95 \%$ by GC analysis. $n$-Alkanes were obtained from Sigma-Aldrich.

\section{Lures and Traps}

Lures used in field trials were prepared by polypropylene pipette tips $(1 \mathrm{~mL})$ with a 0.2 mm aperture (Fountain et al. 2014). Certain amounts of HA, HB and 4-OHE dissolved in sunflower oil (100 $\mu \mathrm{L}$; food-grade) were added to a cellulose acetate cigarette filter $(14 \times 6$ $\mathrm{mm})$, and then placed in a pipette tip (1mL, Fisher Scientific) with a $0.2 \mathrm{~mm}$ aperture. Tips were then sealed with a Teflon-lined crimp seal and wrapped in duct tape. Considering the poor flight ability of $H$. minutus, white delta traps with sticky floors $(28 \times 20 \mathrm{~cm}$, Pherobio Technology Co. Ltd. Beijing) were set on the lawn directly.

\section{Field Trapping}

The field trials were carried out in turfgrass (Festca arundinacea) in Institute of Plant Protection $\left(40^{\circ} 01^{\prime} 18.50^{\prime \prime} \mathrm{N}, 116^{\circ} 16^{\prime} 44.26^{\prime \prime} \mathrm{E}\right)$, Beijing. During our experiment period, the turfgrass was mowed every two weeks, and no pesticides were sprayed. Traps were randomly designed with at least $10 \mathrm{~m}$ between adjacent traps. Five duplicates were conducted for each treatment. We examined traps at 10:00 am every day, and replaced sticky inserts simultaneously. After each examination, traps were randomly rearranged to minimize positional effects. Mean daily trap captures were used as indicator for field activity. Trap catch data were analyzed by one-way analysis of variance (ANOVA) followed by Tukey's 
honestly significant differences (HSD) test. A significance level of 0.05 was used for statistical analysis.

Field experiment 1 aimed to verify the existence of sex pheromone in $H$. minutus by using live females from July 15 to 22, 2014. Five virgin females caged in a glass vial (diam 2 $\mathrm{cm}$; height $4.5 \mathrm{~cm}$ ) with gauze were used as lures. During experiments, sunset usually occurred at $\sim 19: 00$, and it get dark at $\sim 20: 00$. To determine when H. minutus mated, treatments were conducted in three successive time periods: 5:00-12:00, 13:00-19:00 and 20:00-4:00(at night). The live bugs and their foods were refreshed at 13:00. Field experiment 2 investigated the attractiveness of candidate components from August 11 to 18, 2014. Treatments were single HA, HB, 4-OHE, their binary and ternary blends, caged virgin females and control (100mg sunflower oil). Experiment 3 optimized the ratio by varying the amounts of one of HA, HB and 4-OHE each time from August 18 to September 8, 2014. Experiment 4 assessed the dose-attractiveness of HA, HB and 4-OHE at the ratio of 1:16:10 from August 8 to 15 , 2015. Experiment 5 was carried out to verify the long-term attractiveness of artificial lures. Lures were prepared by adding $13.5 \mathrm{mg}$ ternary blends of HA, $\mathrm{HB}$ and 4-OHE at the ratio of 1:16:10. Five traps with lures and five unbaited traps (as blank control) were set on the same lawn. Trap captures were recorded weekly from 11 August to 22 September, 2015. 


\section{Results}

\section{Preliminary Field Experiments}

During all experiments, the mean captures of control traps baited with turfgrass in vials were less than 0.5 per replicate for both sexes. In experiment 1 , we tested the mating peak of H. minutus in field. Fig.1 showed that significantly more H. minutus males were trapped by virgin females at night (Fig. $1 . F_{2,10}=526.6, P<0.001$ ), indicating that $H$. minutus copulate in nocturnal rather than diurnal. Captures of H. minutus females were no significant differences among all periods (Fig.1, $F_{2,10}=0.081, P=0.922$ ), and the mean number of captures was no more than $0.5 /$ trap/day.

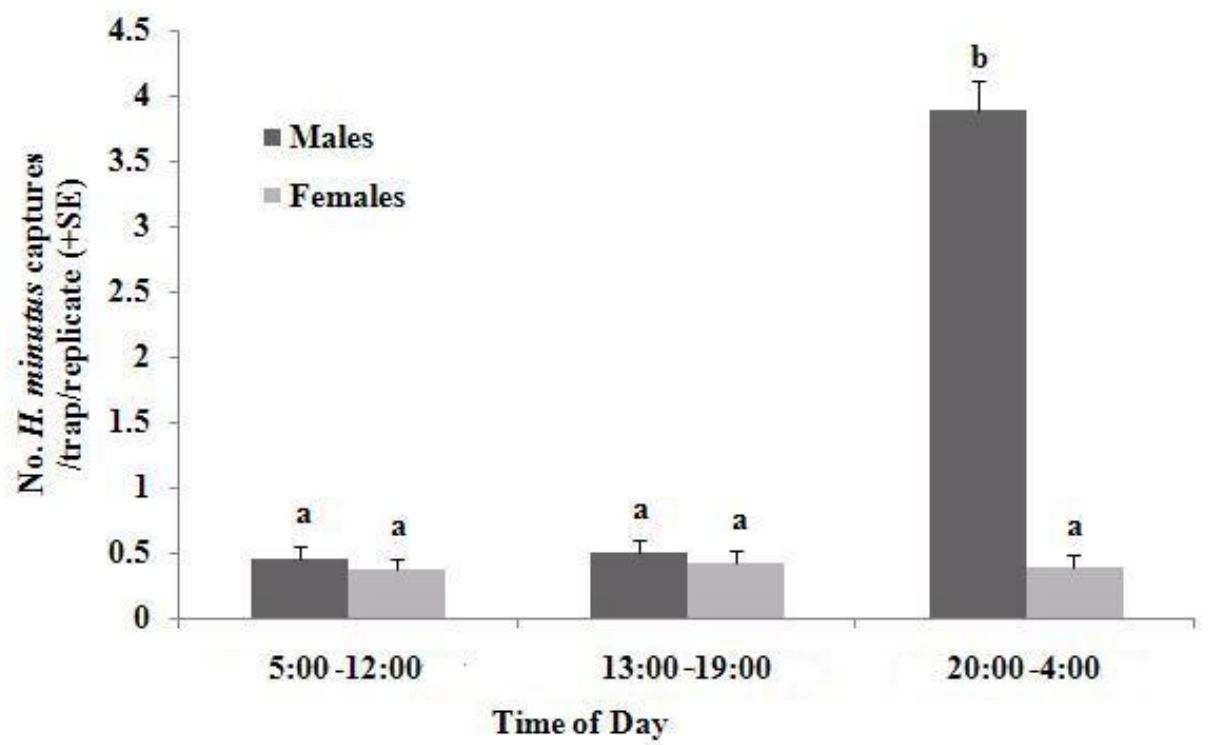

Fig. 1. Captures of Halticus minutus in delta traps baited with virgin females during three periods of the day (July 15 to 22, 2014). Bars with the same letter are not significantly different $(p>0.05)$. 


\section{Chemicals Identification}

Volatiles were collected during scotophase because H. minutus females emitted sex pheromone for copulation at night. GC-MS analysis revealed that three major components were identified as $\mathrm{HA}, \mathrm{HB}$ and 4-OHE in the volatiles of virgin $H$. minutus females (Table 1). The retention induces and the mass spectrums of these components were in accordance with those of commercial compounds. HB and 4-OHE were dominant in the volatiles of females, whereas HB was the most abundant component for males. In female volatiles, the ratio of HA, $\mathrm{HB}$, and 4-OHE was analyzed as 4:100:40, and the amount of $\mathrm{HB}$ was estimated to be $2483 \pm 987 \mathrm{ng}$ per insect $(N=10)$. Several trace compounds were also detected in adult volatiles, including hexanal, $(E)$-2-hexenal, hexanol, $(E)$-2-hexenyl acetate and pentyl butyrate (Table 1, Fig. S1).

Table 1. Analysis of volatiles collected from Halticus minutus adults (Mean \pm SD)

\begin{tabular}{ccccc}
\hline \multirow{2}{*}{ Compounds } & \multicolumn{2}{c}{ Retention indice $^{1}$} & \multicolumn{2}{c}{ Amounts (ng/bug) $)^{2}$} \\
\cline { 2 - 5 } & DB-wax & DB-5 & Female & Male \\
\hline Hexanal & 1069 & 803 & $45 \pm 21$ & $39 \pm 18$ \\
(E)-2-Hexenal & 1203 & 846 & $51 \pm 32$ & $46 \pm 21$ \\
Hexyl acetate & 1260 & 996 & $102 \pm 38$ & $59 \pm 28$ \\
Pentyl butyrate & 1310 & 1091 & $32 \pm 13$ & $\mathrm{ND}^{3}$ \\
(E)-2-Hexenyl acetate & 1335 & 1005 & $18 \pm 7$ & $11 \pm 4$ \\
Hexanol & 1352 & 871 & $53 \pm 20$ & $36 \pm 15$ \\
Hexyl butyrate & 1419 & 1190 & $2483 \pm 787$ & $2638 \pm 825$ \\
(E)-4-Oxo-2-hexenal & 1595 & 956 & $988 \pm 355$ & $226 \pm 90$ \\
$(E)$-2-Hexenyl butyrate & 1462 & 1201 & $\mathrm{ND}^{3}$ & $13 \pm 6$ \\
\hline
\end{tabular}

${ }^{1}$ Retention indices calculated according to the retention times of tested compounds and $n$-alkanes. ${ }^{2}$ The amounts of released volatiles were calculated by the standard compounds based on the peak 
areas.

${ }^{3} \mathrm{ND}=$ not detected.

\section{EAG Analysis}

During our previous EAG tests, only three major components $\left(10 \mu \mathrm{g} \cdot \mu \mathrm{L}^{-1}\right)$ elicited significant responses of antennae of $H$. minutus males (Unpublished data). Thus, we further tested the dose-response of the major compounds. Results showed that the EAG values of males increased significantly as the increases of HA, HB and 4-OHE by ten-fold each time. However, the dose-response of females was not obvious (Fig. 2), indicating that these compounds were the potential candidates of sexual communication components in $H$. minutus.

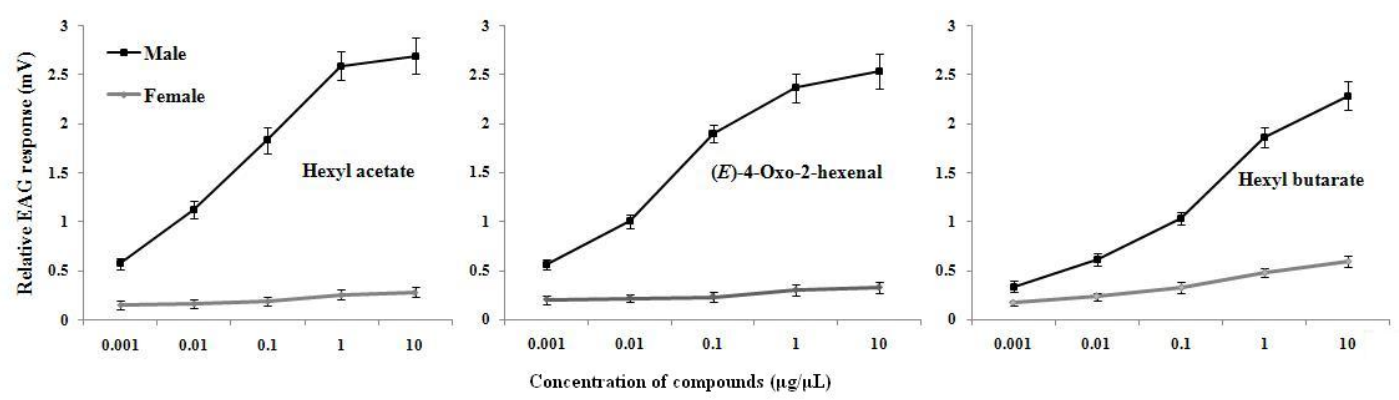

Fig. 2. Dose-dependent EAG response curves of both sexes to sex pheromone components.

\section{Field Trials}

While traps baited with single or binary of $\mathrm{HA}, \mathrm{HB}$ and 4-OHE caught as low H. minutus males as control traps, the ternary blend significantly trapped more males than that of virgin 
females (Fig.3, $F_{8,32}=126.4, P<0.001$ ). We conclude that HA, HB and 4-OHE should be essential components of $H$. minutus sex pheromone.

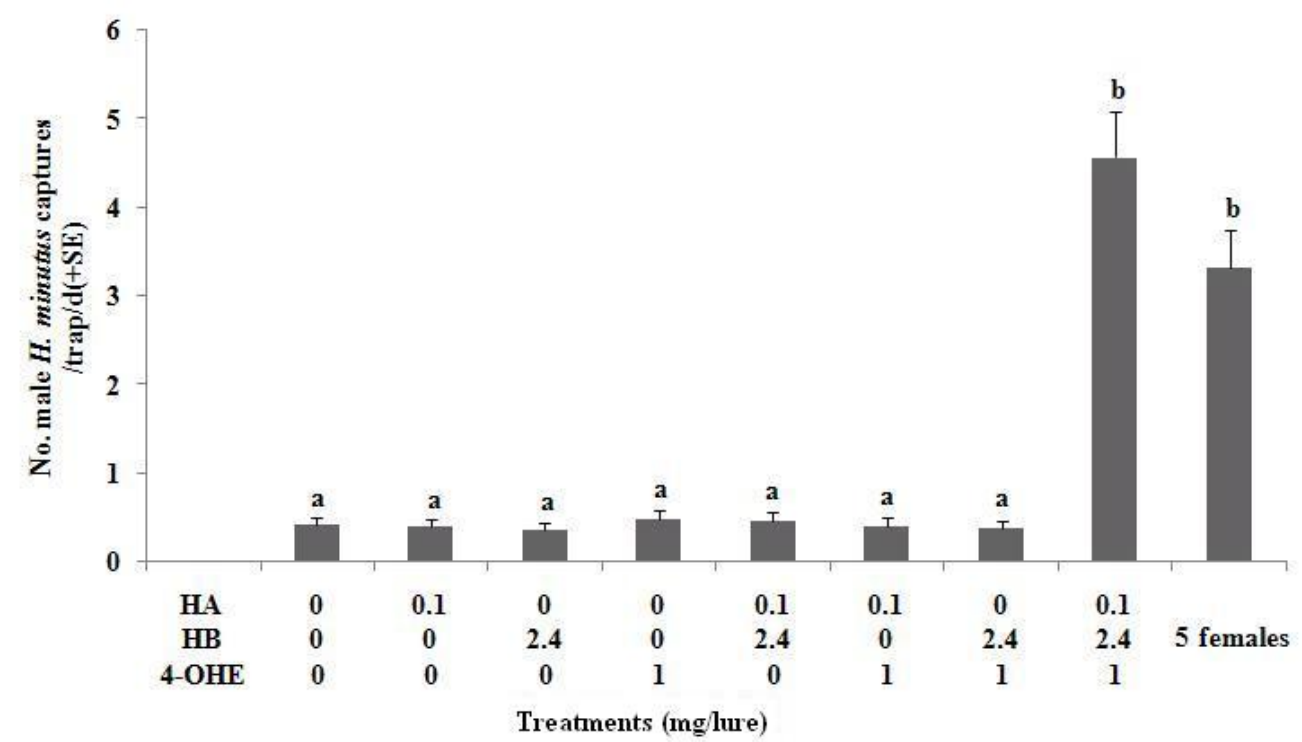

Fig. 3. Captures of Halticus minutus males in traps baited with hexyl acetate (HA), hexyl butyrate (HB), and (E)-4-oxo-2-hexenal (4-OHE), their binary blends and ternary blends and caged virgin females (August 11 to 18,2014$)$. Bars with the same letter are not significantly different $(p>0.05)$.

To optimize the ratio of HA, HB and 4-OHE, lures were prepared with the variable amounts of one component and constant amounts of the other two. Amounts of each component used in the trials were based on field experiment 2. The results showed that captures of $H$. minutus males were heavily dependent on the ratio and dose of three components (Fig.4). When the amount of HA ranged from 0.075 to $0.15 \mathrm{mg} /$ tube, significantly more males were caught $\left(F_{4,16}=21.2, P<0.001\right)$, and lures loaded $0.1 \mathrm{mg}$ HA trapped the most bugs (Fig.4A, mean captures $4.23 \pm 0.4$ /trap/day). Amounts of $\mathrm{HB}$ affected the captures 
significantly, and 1.6mg of $\mathrm{HB}$ addition was evaluated as the optimum (Fig.4B, mean captures $7.54 \pm 0.69$ /trap/day). Higher HB addition decreased the captures sharply, and when double dose of HB were added, the captures would be completely inhibited (Fig.4B, $\left.F_{4,16}=53.3, P<0.001\right)$. Similarly, lures loaded $1 \mathrm{mg}$ 4-OHE trapped the most $H$. minutus males (Fig.4C, $F_{4,16}=106.1, P<0.001$ ), and extra 4-OHE reduced the number of captures (Fig.4C, mean captures $4.71 \pm 0.43$ /trap/day). As such, the optimum ratio of HA to HB to 4-OHE was determined to be $1: 16: 10$.

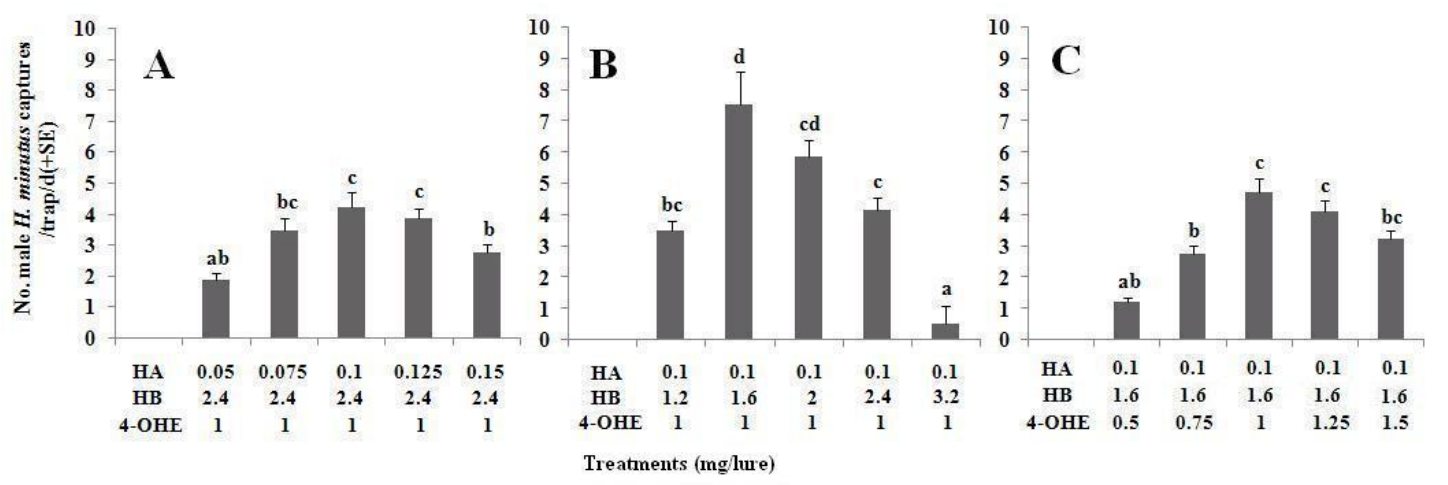

Fig. 4. Captures of Halticus minutus males in traps baited with different ratio of HA, HB, and 4-OHE

(August 18 to September 8, 2014). A: from August 18 to 25; B: from August 25 to September 1; C:

from September 1 to 8 . Bars with the same letter are not significantly different $(p>0.05)$.

At optimum ratio, field experiment 4 was conducted to evaluate the dose-attractiveness with the total amounts ranged from 0.27 to $27 \mathrm{mg}$ per lure. Fig.5 showed that the attractiveness of lures significantly depended on the addition amounts of the tertiary blend of HA, HB and 4-OHE (Fig. 5, $F_{5,20}=280.6, P<0.001$ ). $13.5 \mathrm{mg}$ of pheromone was tested as 
optimum, because more addition could not increase the captures. Perhaps, high-dose of pheromone could extend the working life, which was determined by the capacity of the dispenser (Fountain et al. 2014). The long-term attractiveness of pipette tip lures were also evaluated, and Fig.6 showed that significantly more $H$. minutus males were caught in traps baited with lures than unbaited control during experiment period. Although the number of captures began to decrease at 6 th weeks (Fig. $6, F_{6,24}=5.63, P<0.01$ ), the effective duration of pipette tip lures were long-enough to use for monitoring or controlling.

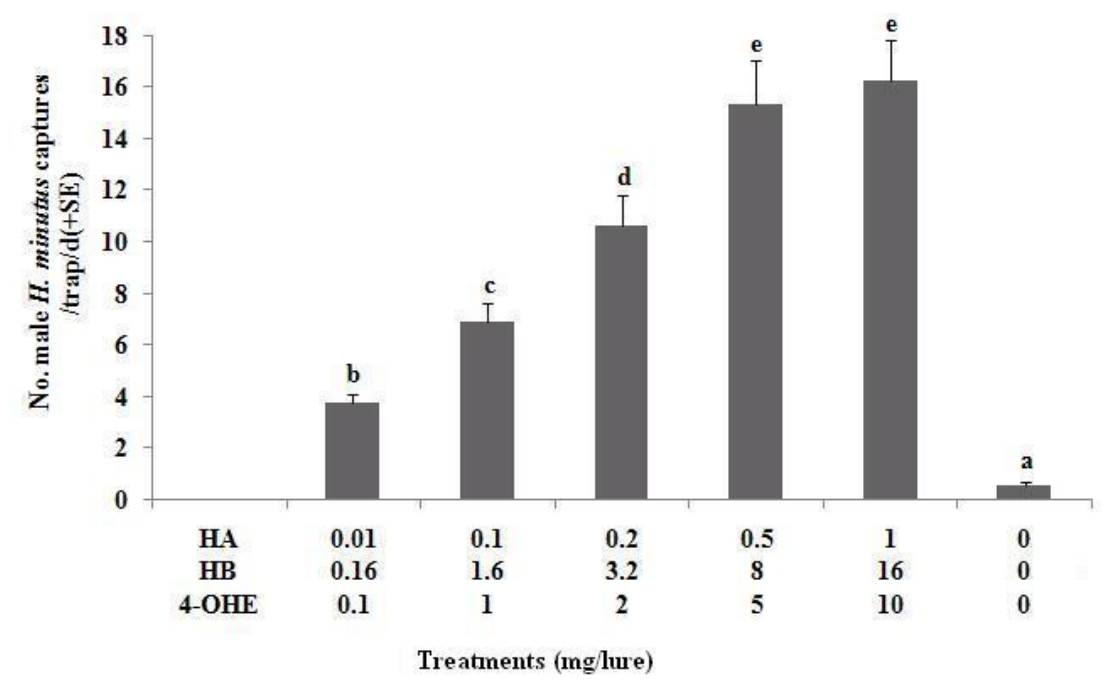

Fig. 5. Dose-dependent captures of Halticus minutus males in traps baited with HA, HB, and 4-OHE at the ratio of 1:16:10 (September 8 to 15, 2014). Bars with the same letter are not significantly different $(p>0.05)$. 


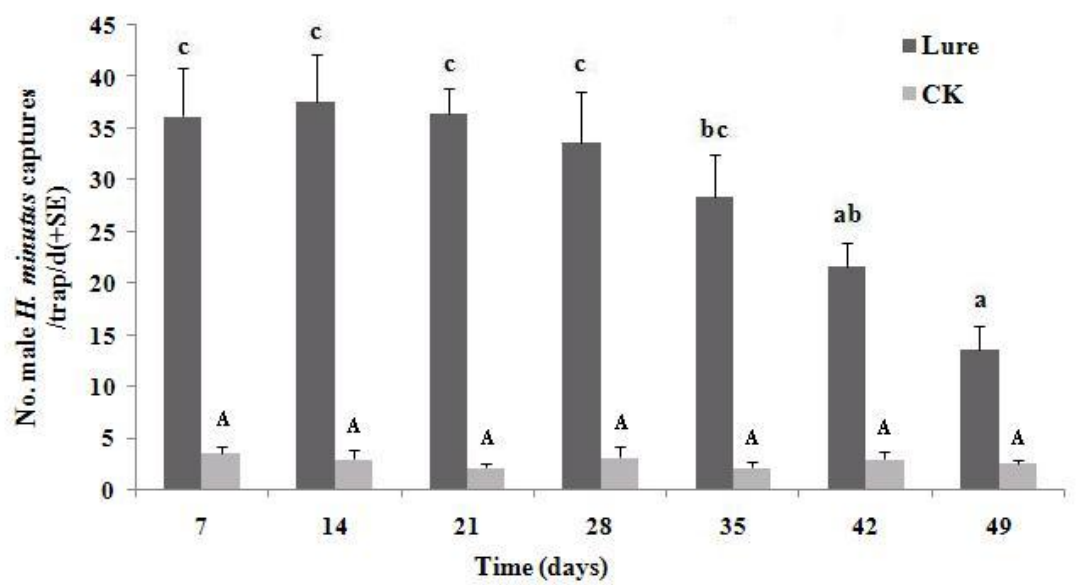

Fig. 6. Captures of Halticus minutus males in traps baited with $13.5 \mathrm{mg}$ of HA, HB, and 4-OHE at the ratio of 1:16:10 (August 11 to September 22, 2015). Bars with the same letter are not significantly different $(p>0.05)$.

Because delta traps were placed on the surface of turf directly, non-target insects were also caught on the viscous inserts, including spiders, ants, aphis, leafhoppers, flies and moths. However, the captures had no significant differences between treatments and control (unpublished data), suggesting these insects were caught by accident. 


\section{Discussion}

To our knowledge, there have been no reports yet regarding the chemical ecology of genus Halticus. In our present study, three major components of $H$. minutus adults were identified as HA, HB and 4-OHE, which could elicit strong responses to male antennae. Their ternary blend was identified as sex pheromone of $H$. minutus due to trapping significantly more males in fields.HA, functioned as a sex pheromone component, had been detected in plant bugs, e.g Phytocoris species (Millar and Rice, 1998; Millar et al., 1997; Zhang and Aldrich, 2003a, 2008). In addition to acting as sex pheromone, HA and other volatiles from Phytocoris calli Knight were believed to contribute to maintaining reproductive isolation of Phytocoris spp. (Zhang and Millar, 2008). HA was also determined as alarm pheromone (Blatt et al., 1998; Leal et al., 1994) and defensive compounds (Prudic et al., 2008) of species in family Coreidae.

HB and 4-OHE were also detected among various plant bugs, not only from Metathoracic Scent Glands (MSG) but also from emitted volatiles. They functioned as sex pheromone, defensive secretion, alarm pheromone or anti-sex pheromone. (Byers, 2006; Byers et al., 2013; Drijfhout et al., 2001; Fountain et al., 2014; Innocenzi et al., 2004, 2005; Oku and Yasuda, 2010; Wardle et al., 2003; Zhang and Aldrich, 2003b). Recently, HB and 4-OHE have been identified as key components of sex pheromone of Lygus spp. (Fountain et al. 2014), and estimated to play an important role in the reproductive isolation (Byers et al. 2013). One of our recent study showed that HB and 4-OHE composed the sex pheromone of Adelphocoris spp. (Zhang et al., 2015; Zhang et al., 2016). When L. Hesperus and L. lineolaris were disturbed or attacked, they released more HB, 4-OHE and other volatiles as intraspecific alarm pheromone or as chemical weapons against predators (Byers, 2006; Wardle et al., 2003). In Phytocoris difficilis (Zhang and Aldrich, 2003b), Lygocoris pabulinus (Groot et al. 2001) and Apolygus spinolae (Yang et al., 2014, 2015), new-mated females released $\mathrm{HB}$ to prevent more copulations through interruption of sex communication, indicating that HB could modulate copulation rhythm. This cluster of study demonstrated that HB and other volatiles might play multiple roles in some Mirid species. In terms of the study 
on H.minutus, we were thinking that sex pheromone compounds also functioned as alarm pheromone or defensive secretion, because we obviously smelled the specific odor of ester (such as HB) when we collected this insect by net-sweeping. Furthermore, this assumption was verified by further GC-MS analyses: more HA, HB and 4-OHE, especially HB, emitted from disturbed both sexes of $H$. minutus (unpublished data). The discrepancy, between analytic ratio from volatiles and optimized ratio in field test, might make sense as the sensitive insects were disturbed by slight vibration during collecting volatiles.

Rubber septa were usually used as pheromone dispenser for plant bugs (Millar et al. 1997; Zhang and Aldrich 2003a). Although sex pheromones of Mirid bugs would exhaust from septa only a few days (Innocenzi et al., 2004), septa were capable for preliminary screening of candidate components in short interval experiments (Yasuda et al., 2008, 2013). In recent years, more kinds of pheromone carriers were developed to improve the longevity and attractiveness of lures for plant bugs. Yasuda et al. (2013) developed high-density polyethylene tubes as pheromone dispensers of Stenotus rubrovittatus, the validity period was more than 28 days. And, they demonstrated that the ratio of esters and 4-OHE emitted from dispensers was similar to the blend initially loaded. Yang et al. (2014) used the same type of dispenser and caught significantly more Apolygus spinolae male in a relative long-term period. However, lower captures indicated that neither rubber septa nor high-density polyethylene tubes were suitable as the pheromone dispenser of $H$. minutus. A novel kind of pheromone lure for mirid, which could release ester and 4-OHE slowly, was developed by Fountain et al. (2014). We also employed pipette tips as long-term dispensers during field experiments. Lures loaded with HA, HB and 4-OHE (1mg, 16mg and 10mg, respectively) trapped significantly more $H$. minutus males in more than 5 weeks. During experiment period, there are no significant differences in the captures of blank control (were trapped by accident) (Fig. 6), suggesting populations of $H$. minutus did not decline. Meanwhile, we did not notice significant population declines when we collected bugs in another turfgrass which was about one kilometer distant from experiment site (Unpublished data).These results indicate the optimal longevity of lures should be $\sim 5$ weeks. Finally, we believe this kind of lures might be useful as long-term dispensers in control of plant bugs. 
Our results, for the first time, revealed that the sex pheromone of $H$. minutus was composed of $\mathrm{HA}, \mathrm{HB}$ and 4-OHE at ratio of 1:16:10. Traps with artificial lures caught significantly more males in 5 weeks period. Further studies should be conducted to determine the actual functions of $\mathrm{HB}$ and minor components. In addition, more efficient and convenient traps need to be designed and developed.

Acknowledgments - We thank Prof David Stanley (Biological Control of Insects Research Laboratory, Agricultural Research Service, USDA) for assistance in preparation of the manuscript. This work was funded by National Basic Research Program of China (973 Program: 2012CB11410) and the National Natural Science Foundation of China (3130914 and 31321004). 


\section{References}

Amalin, D.M., Vasquez, E.A., 1993. A handbook on Philippine sweet potato pests and their natural enemies. International Potato Center (CIP), Los Baños, Philippines. 82.

Blatt, S.E., Borden, J.H., Pierce, H.D., Gries, R., Gries, G., 1998. Alarm pheromone system of the western conifer seed bug, Leptoglossus occidentalis. J. Chem. Ecol. 24(6), 1013-1031.

Byers, J.A., 2006. Production and predator-induced release of volatile chemicals by the plant bug Lygus hesperus. J. Chem. Ecol. 32(10), 2205-2218.

Byers, J.A., Fefer, D., Levi-Zada, A., 2013. Sex pheromone component ratios and mating isolation among three Lygus plant bug species of North America. Naturwissenschaften. 100(12), 1115-1123.

Cassis, G., Schuh, R.T., 2012. Systematics, biodiversity, biogeography, and host associations of the Miridae (Insecta: Hemiptera: Heteroptera: Cimicomorpha). Annu. Rev. Entomol. 57, $377-404$

Drijfhout, F.P., Groot, A.T., 2001. Close-Range Attraction in Lygocoris pabulinus (L.). J. Chem. Ecol. 27(6), 1133-1149.

Fountain, M., Jåstad, G., Hall, D., Douglas, P,, Farman, D., Cross, J., 2014. Further studies on sex pheromones of female Lygus and related bugs: development of effective lures and investigation of species-specificity. J. Chem. Ecol. 40(1), 71-83.

Groot, A.T., Drijfhout, F.P., Heijboer, A., van Beek, T.A., Visser, J.H., 2001. Disruption of sexual communication in the mirid bug Lygocoris pabulinus by hexyl butanoate. Agr. Forest Entomol. 3, 49-55. 
Henry, T.J., 1983. The garden fleahopper genus Halticus (Hemiptera: Miridae): resurrection of an old name and key to species of the western Hemisphere. P. Entomol. Soc. Wash. 85(3), 607-611.

Ho, H.Y., Millar, J.G., 2002. Identification, electroantennogram screening, and field bioassays of volatile chemicals from Lygus hesperus Knight (Heteroptera: Miridae). Zool. Stud. 41, 311-320.

Innocenzi, P.J., Hall, D.R., Cross, J.V., Masuh, H., Phythian, S.J., Chittamaru, S., Guarino, S., 2004. Investigation of long-range female sex pheromone of the European tarnished plant bug, Lygus rugulipennis: chemical, electrophysiological, and field studies. J. Chem. Ecol. 30(8), 1509-1529.

Innocenzi, P.J., Hall, D.R., Cross, J.V., Hesketh, H., 2005. Atraction of male European tarnished plant bug, Lygus rugulipennis to components of the female sex pheromone in the field. J. Chem. Ecol., 31(6), 1401-1413.

Kakizaki, M., Sugie, H., 2001. Identification of female sex pheromone of the rice leaf bug, Trigonotylus caelestialium. J. Chem. Ecol. 27(12), 2447-2458.

Leal, W.S., Panizzi, A.R., Niva, C.C., 1994. Alarm pheromone system of leaf-footed bug Leptoglossus zonatus (Heteroptera: Coreidae). J. Chem. Ecol. 20(5), 1209-1216.

Lowor, S.T., Del Socorro, A.P., Gregg, P.C., 2009. Sex pheromones of the green mirid, Creontiades dilutus (Stål) (Hemiptera: Miridae). Int. J. Agric. Res. 4(4), 137-145.

Mahob, R.J., Babin, R., ten Hoopen, G.M., Dibog, L., Hall, D.R., Bilong, C.F., 2011. Field evaluation of synthetic sex pheromone traps for the cocoa mirid Sahlbergella singularis (Hemiptera: Miridae). Pest Manag. Sci. 67(6), 672-676. 
Millar, J.G., Rice, R., 1998. Sex pheromone of the plant bug Phytocoris califonicus (Heteroptera: Hemiptera: Miridae). J. Econ. Entomol. 91, 132-137.

Millar, J.G., Rice, R., Wang, Q., 1997. Sex pheromone of the mirid bug Phytocoris relativus. J. Chem. Ecol. 23, 1743-1754.

Mitchell, P.L., 2004. Heteroptera as vectors of plant pathogens. Neotrop. Entomol. 33(5), $519-545$.

Moreira, J.A., Millar, J.G., 2005. Short and simple syntheses of 4-oxe-(E)-2-hexenal and homologs: pheromone components and defensive compounds of Hemiptera. J. Chem. Ecol. 31(4), 965-968.

Oku, K., Yasuda, T., 2010. Effects of age and mating on female sex attractant pheromone levels in the sorghum plant bug, Stenotus rubrovittatus (Matsumura). J. Chem. Ecol. 36, $548-552$

Prudic, K.L., Noge, K., Becerra, J.X., 2008. Adults and nymphs do not smell the same: the different defensive compounds of the giant mesquite bug (Thasus neocalifornicus: Coreidae). J. Chem. Ecol. 34(6), 734-741.

Sarfo, J.E., 2013. Behavioural responses of cocoa mirids, Sahlbergella singularis Hagl and Distantiella theobroma Dist. (Heteroptera: Miridae), to sex pheromones. Doctoral dissertation, University of Greenwich.

Smith, R.F., Pierce, H.D., Borden, J.H., 1991. Sex pheromone of the mullein bug, Campylomma verbasci (Meyer) (Heteroptera: Miridae). J. Chem. Ecol. 17, 1437-1447.

Sun, L., Xiao, H.J., Gu, S.H., Guo, Y.Y., Liu, Z.W., Zhang, Y.J., 2014. Perception of potential sex pheromones and host-associated volatiles in the cotton plant bug, Adelphocoris fasciaticollis (Hemiptera: Miridae): morphology and electrophysiology. Appl. Entomol. 
Zool. 49, 43-57.

Tong, X.S., Wang, L.S., 1987. Bionomics and control of the fleahopper Halticus minutus Reuter. Acta Entomol. Sin. 30(1), 113-115. (in Chinese)

Wang, Y.B., Wang, J.M., Pan, P.L., Yu, H., Zhang, Z.Y., 2005. The investigation of Festca arundinacea turf insects and their communities in north of Henan province. Chinese Agric. Sci. Bull. 21(5), 323-326. (in Chinese)

Wardle, A.R., Borden, J.H., Pierce, H.D., Gries, R., 2003. Volatile compounds released by disturbed and calm adults of the tarnished plant bug, Lygus lineolaris. J. Chem. Ecol. 29(4), 931-944.

Witzgall, P., Kirsch, P., Cork, A., 2010. Sex pheromones and their impact on pest management. J. Chem. Ecol. 36(1), 80-100.

Xu, X.W., Yin, S.Z., Wang, J.M., Zhang, Z.Y., Wang, Y.B., 2010. Investigation of wide-leafy turf insects and their occurrence tendency in north of henan province. Acta Agric. Jiangxi. 22(8), 99-100. (in Chinese)

Yang, C.Y., Kim, J., Ahn, S.J., Kim, D.H., Cho, M.R., 2014. Identification of the female-produced sex pheromone of the plant bug Apolygus spinolae. J. Chem. Ecol. 40(3), 244-249.

Yang, C.Y., Kim, S.J., Kim, J., Kang, T.J., Ahn, S.J., 2015. Sex pheromones and reproductive isolation in five Mirid species. PloS one, 10(5), e0127051.

Yasuda, T., Mochizuki, F., Yasuda, M., Takeda, A., Higuchi, H., Watanabe, T., Yamashita, M., Fukumoto, T., 2013. Performance of polyethylene tubes as pheromone lures for the sorghum plant bug, Stenotus rubrovittatus (Hemiptera: Heteroptera: Miridae). Appl. 
Entomol. Zool. 48: 325-330.

Yasuda, T., Shigehisa, S., Yuasa, K., Okutani-Akamatsu, Y., Teramoto, N., Watanabe, T., Mochizuki, F., 2008, Sex attractant pheromone of the sorghum plant bug Stenotus rubrovittatus (Matsumura) (Heteroptera: Miridae). Appl. Entomol. Zool. 43 (2), 219-226.

Zhang, Q.H., Aldrich, J.R., 2003a. Pheromones of milkweed bugs (Heteroptera: Lygaeidae) attract wayward plant bugs: Phytocoris mirid sex pheromone. J. Chem. Ecol. 29, 1807-1823.

Zhang, Q.H., Aldrich, J.R., 2003b. Male-produced anti-sex pheromone in a plant bug. Naturwissenschaften. 90, 505-508.

Zhang, Q.H., Aldrich, J.R., 2008. Sex Pheromone of the Plant Bug, Phytocoris calli Knight. J. Chem. Ecol. 34, 719-724.

Zhang, T., Mei, X.D., Zhang, L.X., Wu, K.M., Ning, J., 2015. Identification of female sex pheromone of a plant bug, Adelphocoris fasciaticollis Reuter (Hemiptera: Miridae). J. Appl. Entomol. 139(1-2), 87-93.

Zhang, T., Mei, X.D., Li, Y.F., Zhang, K.X., Wu, K.M., Ning, J., 2015. Sex pheromone of the alfalfa plant bug, Adelphocoris lineolatus. Entomol. Exp. Appl. 156(3), 263-270.

Zhang, Z.L., Zhang, T., Zhang, A.J., Luo, J., Chen, L.Z., Wang, M.Q., Ning, J., Lei, C.L., 2016. Identification and field verification of sex pheromone from the mirid bug, Adelphocoris suturalis. Chemoecology. 26(1), 25-31. 